\title{
Survival and recurrence factors in adult medulloblastoma: The M.D. Anderson Cancer Center experience from 1978 to 1998
}

\author{
Lara J. Kunschner, John Kuttesch, Kenneth Hess, and W.K. Alfred Yung ${ }^{1}$ \\ Departments of Neuro-Oncology (L.J.K., W.K.A.Y.), Biomathematics (K.H.), and Division of Pediatrics (J.K.), \\ The University of Texas M.D. Anderson Cancer Center, Houston, TX 77030
}

Medulloblastoma is a rare adult primary brain tumor for which limited retrospective studies are available to elucidate natural history or to guide therapy. A retrospective chart and imaging review of adult patients (aged $>18$ years) with medulloblastoma was performed to identify survival and prognostic factors. Fifty-seven patients were evaluated at the University of Texas M.D. Anderson Cancer Center from 1978-1998. Statistical analysis of prognostic factors and overall survival was performed for a subgroup of 28 patients who were followed exclusively at our institution from the time of diagnosis until death or last follow-up. These 28 patients had an overall survival of $91 \%$ at 3 years and $84 \%$ at 5 years, whereas median survival was not reached after a median follow-up of 168 weeks (range, 9-602 weeks). Progression-free survival for all patients was $68 \%$ at 3 years and $62 \%$ at 5 years, and was not statistically different between poor- and standard-risk patients. Univariate analysis of clinical features, such as age, sex, extent of local disease, extent of resection, and use of adjuvant chemotherapy, did not identify any prognostic variables for survival among the 28 patients. Patterns of recurrence revealed that the posterior fossa was the most common site $(56 \%)$, followed by bone marrow $(25 \%)$. Adult medulloblastoma appears to have a favorable prognosis after treatment with maximally surgically feasible resection followed by craniospinal irradiation.

Received 14 December 1999, accepted 19 January 2001.

${ }^{1}$ Address correspondence and reprint requests to W.K. Alfred Yung, Professor and Acting Chairman, Department of Neuro-Oncology, Box 100, The University of Texas M.D. Anderson Cancer Center, 1515 Holcombe Blvd., Houston, TX 77030.

${ }^{2}$ Abbreviations used are as follows: $\mathrm{Cl}$, confidence interval; CSF, cerebrospinal fluid; MDACC, University of Texas M.D. Anderson Cancer Center.
Optimal treatment remains to be clarified, as both standard-risk and poor-risk patients have prolonged diseasefree survival. The marked difference between survival and progression-free survival suggests that salvage therapy, usually with combination chemotherapy in this cohort of patients, is of benefit. More formal analysis of the survival benefit was not possible, however, because of the small number of patients treated at recurrence with any one therapeutic regimen. Neuro-Oncology 3, 167-173, 2001 (Posted to Neuro-Oncology [serial online], Doc. 99-058, May 15, 2001. URL <neuro-oncology.mc. duke.edu>)

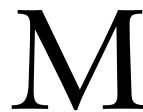
edulloblastoma represents an uncommon adult tumor comprising less than $1 \%$ of adult primary neoplasms (Bloom and Bessel, 1990). Incidence rates are approximately 0.5 per million (Giordana et al., 1999) with a progressive decrease in incidence with advancing age. The Surveillance, Epidemiology and End Results Database lists only 186 adult cases. Several retrospective studies have offered insight into the natural history of the disease, but because of the paucity of patients, prospective treatment trials have not been undertaken exclusively for adult patients. True survival rates and prognostic factors remain unclear because the largest retrospective series of adult cases, which reported an overall 5 -year survival of $70 \%$, included only 156 patients culled from 13 separate institutions over a period of 15 years (Carrie et al., 1994). The patients included in these retrospective reports were treated according to a wide variety of therapy protocols, rendering it difficult to use those reports to assess overall prognosis and prognostic factors.

Adult patients with medulloblastoma are commonly given therapies based on therapy developed for children with similarly staged disease at diagnosis. The question of whether adult medulloblastoma behaves differently from childhood medulloblastoma, however, remains relatively 
unanswered. Pathologic studies suggest phenotypic differences between childhood and adult medulloblastoma (Giordana et al., 1997) that may or may not portend a difference in prognosis. Clinical characteristics have shown some variation between children and adults, for example, a greater proportion of cerebellar hemisphere tumors present in adults. It remains unclear whether advancing age beyond infancy, which is a known poor prognostic factor, has a relationship to survival.

We describe here a series of patients with adult medulloblastoma seen at the University of Texas MDACC ${ }^{2}$ and review their clinical and therapeutic features. A subset of patients followed at MDACC exclusively are examined in detail for survival, progression-free survival, and possible prognostic factors to attempt to clarify these features in a large cohort of patients for which the retrospective data were complete and initial treatment at diagnosis was fairly uniform.

\section{Patients and Methods}

Fifty-seven patients, older than 18 years and with a diagnosis of medulloblastoma, were seen and examined in the Brain Tumor Center at MDACC from 1978 until 1998. Twenty-eight of these patients were seen at the time of the initial diagnosis, or immediately after initial resection, and subsequently received treatment and follow-up at MDACC until the time of death or most recent follow-up. Ten patients were first seen at MDACC at the time of their first tumor recurrence. The remaining 19 patients were seen at MDACC either after two or more recurrences or as a consultation during a period of disease remission. A retrospective review of the patients' medical records supplied the information for this analysis. All living patients had follow-up within 4 months of data analysis.

The records of all 57 patients were used to develop a descriptive profile of medulloblastoma in this adult age group. The subgroup of 28 patients who were seen first at time of diagnosis and who subsequently remained at MDACC for the duration of therapy and follow-up were analyzed for survival and prognostic factors. The remaining 29 patients were excluded from statistical analysis of overall and event-free survival to limit confounding referral bias. The clinical features of the disease in these 29 patients are included in this report to highlight the variation in presentation and treatment of medulloblastoma in the community.

Disease stage was established using the surgical report, radiographic reports, and laboratory reports of CSF findings. The extent of initial surgical resection was determined by review of the surgical report and the postoperative CT scan or MRI. Initial tumor extent was determined by complete brain and spinal imaging and CSF analysis in 27 of the total $57(47 \%)$ patients and in 21 of the $28(75 \%)$ patients treated from initial diagnosis at MDACC. Thirty-nine of 57 patients $(68 \%)$ had either CSF analysis or spine imaging to assess spinal dissemination of tumor. All patients' pathology slides were reviewed by one of three neuropathologists at MDACC.

Patients were assigned to either standard- or poor-risk groups based on the extent of surgical resection and evi- dence for dissemination of tumor outside the posterior fossa at the time of diagnosis. Current accepted staging criteria assign patients with greater than $1.5 \mathrm{~cm}^{2}$ postoperative residual tumor, evidence of CNS dissemination, or extra-neural metastatic disease to the poor-risk category (Packer et al., 1994). Because postoperative tumor measurements on CT or MRI were not routinely available, the patients were stratified to poor risk if there was a radiographic or surgical report of a subtotal resection or biopsy or any other feature of a poor-risk medulloblastoma. The standard-risk designation, for our purposes, included those patients with a reported gross total resection $\left(<1.5 \mathrm{~cm}^{2}\right.$ residual $)$ and no evidence of metastatic tumor outside the posterior fossa on both CSF analysis and complete spine imaging. Systemic metastasis was not routinely looked for at diagnosis, unless the patient had suggestive symptomatology.

Overall survival was determined from the time of diagnosis until death or last follow-up. Tumor progression was defined as $>25 \%$ bidimensional increase in enhancing measurable disease or development of a new locus of tumor, including metastasis outside the CNS. Progression-free survival was measured from the date of surgical resection until the date of confirmed tumor progression by imaging or by death if no progression had been confirmed prior to death. Estimates of survival and progression-free survival were computed using the Kaplan-Meier method. CIs were then calculated. Due to small sample size, univariate analysis, but not multivariate analysis, was performed for multiple variables with regard to impact on overall survival and progressionfree survival.

\section{Results}

\section{Patient Characteristics and Therapy}

Patient characteristics for the subset of 28 patients treated exclusively at MDACC, compared with the entire group of patients, are listed in Table 1. For patients among the entire group of 57 patients, the median age at diagnosis was 26 years (range, 18-57 years). There was a slight predominance of male patients, with $34(60 \%)$ male and $23(40 \%)$ female. Median duration of symptoms was 2 months (range, 1 week to 12 months) prior to diagnosis. Table 2 highlights the presenting symptoms recalled by patients at the time they presented at MDACC. The most common symptom was headache, present in $83 \%$ of patients, followed by nausea and vomiting in $43 \%$ of patients. The remaining symptoms reflect the posterior fossa location of the primary tumor. Thirteen of 57 (23\%) patients presented with symptomatic obstructive hydrocephalus that necessitated placement of a ventriculoperitoneal shunt at the time of or shortly after the initial resection. The medulloblastoma location was centered in the cerebellar vermis in 19 of 57 (33\%); was in the left or right cerebellar hemisphere in 28 of $57(50 \%)$, with an equal number on either side; and could not be specified due to extensive disease in 10 of $57(17 \%)$. Pathology revealed classical medulloblastoma in 41 of $57(72 \%)$ and desmoplastic medulloblastoma in 16 of $57(28 \%)$ patients. 
Table 1. Characteristics of the patient cohort treated solely at MDACC

\begin{tabular}{|c|c|c|c|}
\hline Characteristic & No. of patients $(n=28)$ & Poor-risk patients $(n=16)$ & Standard-risk patients $(n=12)$ \\
\hline \multicolumn{4}{|l|}{ Sex } \\
\hline Male & $17(61)$ & $9(56)$ & $8(66)$ \\
\hline Female & $11(39)$ & $7(44)$ & $4(34)$ \\
\hline Age (years): median (range) & $30.5(19-45)$ & $29(19-45)$ & $31(19-41)$ \\
\hline \multicolumn{4}{|l|}{ Extent of resection } \\
\hline GTR & $15(54)$ & $3(19)$ & $12(100)$ \\
\hline STR & $12(43)$ & $12(75)$ & 0 \\
\hline $\mathrm{BX}$ & $1(3)$ & $1(8)$ & 0 \\
\hline \multicolumn{4}{|l|}{ Metastasis } \\
\hline None identified & $25(89)$ & $13(81)$ & 0 \\
\hline Positive CSF cytology & $2(7)$ & $2(13)$ & 0 \\
\hline Nodular subarachnoid masses & $1(3)$ & $1(6)$ & 0 \\
\hline Extraneural metastasis & $0(0)$ & & \\
\hline \multicolumn{4}{|l|}{ Pathology } \\
\hline Classical & $21(75)$ & $12(75)$ & $9(75)$ \\
\hline Desmoplastic & $7(25)$ & $4(25)$ & $3(25)$ \\
\hline \multicolumn{4}{|l|}{ Adjuvant chemotherapy } \\
\hline None & $22(78)$ & $10(63)$ & $12(100)$ \\
\hline TPDCV & $1(3)$ & $1(8)$ & 0 \\
\hline CVP & $4(14)$ & $4(25)$ & 0 \\
\hline MMOPP & $1(3)$ & $1(8)$ & 0 \\
\hline \multicolumn{4}{|l|}{ Ventriculo-peritoneal shunt } \\
\hline Yes & $5(18)$ & $5(31)$ & 0 \\
\hline No & $23(82)$ & $9(56)$ & $12(100)$ \\
\hline
\end{tabular}

Abbreviations: GTR, gross total resection; STR, subtotal resection; BX, biopsy; TPDCV, thioguanine, procarbazine, dibromodulcitol, $N$-2 (chloroethyl)- $N$-cyclohexyl- $N$-nitrosourea, and vincristine; CVP, cyclophosphamide, VP-16, and cisplatin; MMOPP, methotrexate, nitrogen mustard, vincristine, prednisone, and procarbazine.

Numbers in parentheses, with the exception of age range, are \% of total.

All patients underwent surgery, and diagnosis was established from the resection of the primary lesion. Gross total resection was achieved in $25(44 \%)$, subtotal resection in $31(54 \%)$, and biopsy in $1(2 \%)$. All patients received craniospinal radiotherapy, except for 2 patients who received whole-brain radiation alone in the early 1980s at local facilities. A posterior fossa radiotherapy boost was given to 46 patients $(81 \%)$. Those patients who did not receive a boost presented largely in the late 1970s and early 1980s.

\section{Pattern of Treatment Failure}

At the time of last follow-up, 32 of 57 patients had developed a first recurrence. The most common site of recurrence was within the posterior fossa in $56 \%$ of patients, followed by bone marrow or gross bony metastasis in $25 \%$ (Table 3 ). Additional sites of recurrence were the leptomeninges (nodular or microscopic disease) $(15 \%)$ and soft tissue $(9 \%)$ involving the chest wall, subcutaneous tissue in the neck, lung, or mediastinum. Several patients developed tumor recurrence at multiple sites simultaneously as shown in Table 3 . Fifteen of $57(17 \%)$ patients had a ventriculoperitoneal shunt placed at initial resection, and of those patients, 2 developed extraneural metastasis, 1 solely to the bone marrow. Six of the $15(40 \%)$ remained free from progression, $715(44 \%)$ developed isolated posterior fossa recurrence, and $1(6 \%)$ developed leptomeningeal dis- seminated metastasis concurrent with bone marrow involvement. At the time of recurrence, multiple different chemotherapeutic regimens were used. Details of therapy for the 28 patients treated at MDACC in the survival analysis are presented in Table 4. Analysis for treatment response to any particular regimen at the time of recurrence was limited due to the wide variety of agents and treatment schedules used over the span of 20 years in this small cohort.

Table 2. Presenting signs and symptoms of total patients studied for whom clinical data were available

\begin{tabular}{lc} 
Signs and symptoms & No. of patients $(n=53)$ \\
\hline Headache & $44(83)$ \\
Nausea/vomiting & $23(43)$ \\
Gait imbalance & $21(40)$ \\
Dizziness & $12(23)$ \\
Diplopia & $6(11)$ \\
Limb incoordination & $2(4)$ \\
Hemiparesis & $2(4)$ \\
Tinnitus/hearing loss & $2(4)$ \\
Bell's palsy & $1(2)$ \\
Sensory alteration & $1(2)$ \\
Seizure & $1(2)$
\end{tabular}

Numbers in parentheses are percentage of total. 
Table 3. Site of treatment failure at first recurrence

\begin{tabular}{lcc} 
Location & $\begin{array}{c}\text { No. of MDACC } \\
\text { cohort }(n=28)\end{array}$ & $\begin{array}{c}\text { Total no. of } \\
\text { patients }(n=57)\end{array}$ \\
\hline Posterior fossa only & 6 & 15 \\
Bone only & 2 & 7 \\
Posterior fossa and bone & 0 & 1 \\
Leptomeningeal only & 0 & 2 \\
Leptomeningeal and systemic & 1 & 2 \\
Leptomeningeal and & 0 & 2 \\
$\quad$ posterior fossa & & 2 \\
Systemic soft tissue only & 0 & 1 \\
Location undocumented & 0 &
\end{tabular}

\section{Overall Survival and Progression-Free Survival}

Statistical analysis of survival was performed on only the subgroup of 28 patients treated at MDACC and followed exclusively throughout the course of treatment until death or last follow-up if still living. This was done to eliminate selection bias introduced by including patients referred at first or second recurrence, because it was unknown what percentage of diagnosed patients over that time period had died before they could be referred to a tertiary care center for treatment at recurrence. Followup data were complete for patients treated through their entire course at MDACC, which was not the case for several patients treated at other centers as well as at MDACC. Characteristics in this group of 28 patients were similar to those of the entire group (Table 1). Twelve patients were identified as standard risk and 16 patients as poor risk. Only 3 of 16 patients in the poorrisk group had metastatic disease $(\mathrm{M}+)$ at the time of diagnosis: 2 with positive CSF cytology (M1) and 1 with cauda equina nodular masses (M2). For the 28 patients, median follow-up was 168 weeks (range, 9-602 weeks).

Overall survival, shown in Fig. 1, was 96\% (95\% CI, $88 \%-100 \%)$ at 1 year, $91 \%(95 \% \mathrm{CI}, 79 \%-100 \%)$ at 3 years, and $84 \%(95 \% \mathrm{CI}, 69 \%-100 \%)$ at 5 years. Median survival had not been reached, as a sufficient number of deaths had not occurred to achieve this value. Progression-free survival, shown in Fig. 2, was 96\% (95\% CI, $88 \%-100 \%)$ at 1 year, $68 \%(95 \%$ CI, $51 \%-91 \%)$ at

Table 4. Summary of therapy in the MDACC cohort

\begin{tabular}{|c|c|c|c|c|c|}
\hline Patient no. & Initial surgery & Radiation dose & Adjuvant chemo/\# course & Recurrence & Therapy \\
\hline 1 & STR & CS 34.2 Gy/PF 54 Gy & TPDCV 7 courses & Hip/BM & CVP $\times 6$ \\
\hline 2 & STR & CS 30 Gy/PF 56 Gy & - & - & - \\
\hline 3 & GTR & CS 30 Gy/PF 55 Gy & - & Local & - \\
\hline 4 & GTR & CS 36 Gy/PF 57.8 Gy & - & Local & GTR/XRT/CVP \\
\hline 5 & STR & WB 46 Gy/S 30 Gy/PF 56 Gy & - & CSF/BM & MOPP $\times 3$ \\
\hline 6 & STR & CS 36 Gy/PF 54 Gy & - & Local & MOPP $\times 6$ \\
\hline 7 & GTR & CS 24 Gy hyperfraction/PF 48 Gy & - & - & - \\
\hline 8 & STR & CS 24 Gy hyperfraction/PF 48 Gy & - & - & - \\
\hline 9 & STR & CS 30 Gy/PF 72 Gy & - & - & - \\
\hline 10 & GTR & CS 30 Gy/PF 42 Gy & - & - & - \\
\hline 11 & GTR & CS 30 Gy hyperfraction/PF 42 Gy & - & - & - \\
\hline 12 & STR & WB 40 Gy/S 35 Gy & MMOPP $\times 6$ & - & - \\
\hline 13 & GTR & WB 41.5 Gy/S 34 Gy/PF 54 Gy & - & - & - \\
\hline 14 & Biopsy & CS 35 Gy/PF and cauda 55 Gy & CVP $\times 4$ & - & - \\
\hline 15 & GTR & CS 30 Gy & CVP $\times 5$ & - & - \\
\hline 16 & GTR & CS 35 Gy/PF 60 Gy & - & - & - \\
\hline 17 & GTR & CS 36 Gy & - & - & - \\
\hline 18 & GTR & CS 35 Gy/PF 55 Gy & - & - & - \\
\hline 19 & GTR & CS 34 Gy/PF 54 Gy & - & - & - \\
\hline 20 & STR & CS 30 Gy/PF 55 Gy & - & - & - \\
\hline 21 & STR & CS 30 Gy/PF 56 Gy & CVP $\times 3$ & - & - \\
\hline 22 & GTR & CS 35 Gy/PF 55 Gy & - & - & - \\
\hline 23 & STR & CS 30 Gy/PF 56 Gy & - & - & - \\
\hline 24 & GTR & CS 35 Gy/PF 55 Gy & - & - & - \\
\hline 25 & GTR & CS 35 Gy/PF 55 Gy & - & Posterior fossa & CVP $\times 2$ \\
\hline 26 & GTR & CS 36 Gy/PF 59.8 Gy & - & $\mathrm{BM}$ & $\begin{array}{l}\text { CVP } \times 3 / \text { cytoxan } \\
\text { stem cell transplant }\end{array}$ \\
\hline 27 & STR & WB 40 Gy/S 30.6 Gy/PF 56 Gy & CVP $\times 8$ & Posterior fossa & $?$ \\
\hline 28 & STR & CS 30 Gy/PF 56 Gy & - & - & - \\
\hline
\end{tabular}

Abbreviations: STR, subtotal resection; CS, craniospinal; PF, posterior fossa; TPDCV, thioguanine, procarbazine, dibromodulcitol, CCNU, and vincristine; BM, bone marrow; CVP, cyclophosphamide, VP-16, and cisplatin; GTR, gross total resection; XRT, radiation therapy; WB, whole brain; MOPP, methotrexate, nitrogen mustard, vincristine, prednisone, and procarbazine; S, spine; MMOPP, methotrexate, nitrogen mustard, vincristine, prednisone, and procarbazine; cauda, cauda equina. 


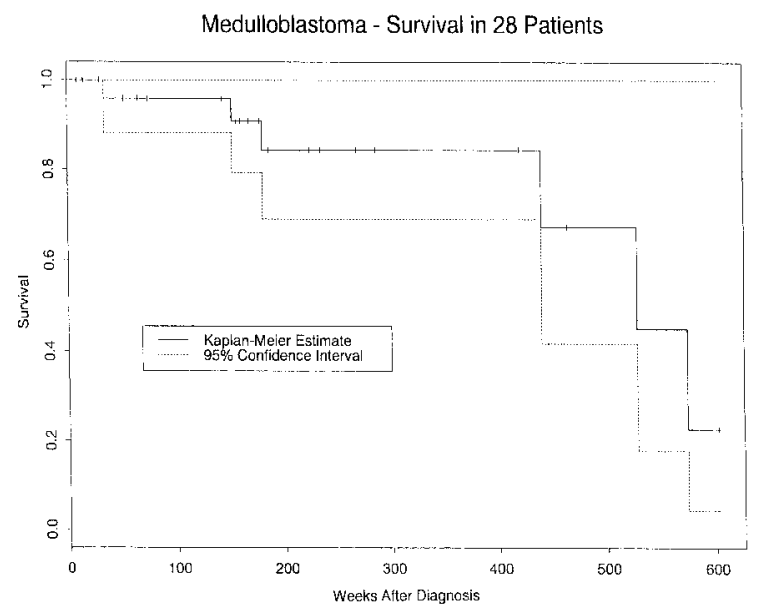

Fig. 1. Kaplan-Meier estimate of overall survival for adult medulloblastoma patients. Survival was measured from date of diagnosis until death or most recent follow-up.

3 years, and $62 \%(95 \% \mathrm{CI}, 44 \%-87 \%)$ at 5 years. Overall survival according to risk group showed a trend toward increased survival at 5 years for standard-risk patients; however, a small total number of deaths occurred in both groups. The median survival in poor-risk patients was 425 weeks (over 8 years), but was not reached in standard-risk patients. The hazard ratio comparing overall survival in poor- versus standard-risk patients was 1.9 , with $P=0.59$, but the $95 \%$ CI extended from 0.2 to 18 . There was only one death in the standardrisk group. Individual analysis of the 3 poor-risk patients with $\mathrm{M}+$ disease revealed overall survival of 34,180 , and $216+$ weeks. Two of the 3 patients with metastatic disease received subtotal resection followed by craniospinal irradiation and adjuvant chemotherapy. One patient received adjuvant cyclophosphamide, VP-16, and cisplatin for 4 courses and remains free of disease after 216 weeks. The other of the 2 patients received adjuvant 6-thioguanine, procarbazine, dibromodulcitol, $\mathrm{N}$-2(chloro-ethyl)- $\mathrm{N}$ cyclohexyl- $N$-nitrosourea, and vincristine for 7 courses, developed recurrence in the hip at 78 weeks, was treated with salvage cyclophosphamide, VP-16, and cisplatin for 6 courses, but later suffered widespread bony metastasis and had a survival after diagnosis of 180 weeks. A third patient presented with cauda equina metastasis (M2) and was treated with craniospinal irradiation alone. This patient survived 34 weeks after diagnosis.

Analysis of traditional prognostic variables for overall and progression-free survival by univariate analysis did not identify prognostic value of age, sex, tumor location, pathologic diagnosis of classical versus desmoplastic medulloblastoma, or extent of initial surgery. Progressionfree survival was not significantly different in standardversus poor-risk patients. Six of $16(38 \%)$ poor-risk patients received adjuvant combination chemotherapy with cyclophosphamide, VP-16, and cisplatin $(n=4)$; 6-thioguanine, procarbazine, dibromodulcitol, $\mathrm{N}$-2 (chloroethyl)-N-cyclohexyl-N-nitrosourea, and vincristine $(n=1)$; or methotrexate, nitrogen mustard, vincristine, prednisone, and procarbazine $(n=1)$. Two of these 6 patients had
M1, 1 had M2, and 3 had M0 disease. No significant difference in overall or progression-free survival was demonstrated between the cohort receiving chemotherapy versus the cohort without chemotherapy.

\section{Discussion}

This retrospective study reflects the clinical experience with treatment of adult medulloblastoma at one institution over a 20-year time period. Patient characteristics are comparable to those reported by several authors for small cohorts of adult patients (Giordana et al., 1999; Noel and Merrer, 1997; Wolff et al., 1998). The findings of previous retrospective studies are supported and expanded by the addition of these patients to the body of data published to date.

Reported 5-year overall survival rates in adult patients treated with megavoltage craniospinal radiation range from $58 \%$ (Le et al., 1997) to 76\% (Bloom and Bessel, 1990; Giordana et al., 1999; Carrie et al., 1994). In the largest series to date, Carrie et al. (1994) reported a 70\% 5 -year overall survival and a $61 \% 5$-year event-free survival for 156 patients drawn from 13 institutions in France. The Surveillance, Epidemiology and End Results Database containing data on 186 adult medulloblastoma patients lists a 5 -year survival of $62 \%$ and a median survival of 10 years (Polednak and Flannery, 1995). Reported median survival has ranged widely from 6 (Le et al., 1997) to 17.6 years (Giordana et al., 1999). Follow-up for our cohort of patients has not been long enough to determine a median survival because so many patients remained alive at the time of analysis, but the overall 5 -year survival rate of $84 \%$ slightly exceeds that reported by other authors (Bloom and Bessel, 1990; Haie et al., 1985; Le et al., 1997; Noel and Merrer, 1997). The somewhat short median followup in this study reflects an increase in incidence in adult medulloblastoma patients who presented for treatment at our institution in the 1990s.

Five-year progression-free survival of $62 \%$ in this series was quite comparable to that reported for adults by Carrie et al. (1994) of $61 \%$. The best outcomes from medulloblastoma reported to date are from Packer et al. (1994), who reported a 5 -year progression-free survival of $85 \%$ for children with medulloblastoma treated with craniospinal and adjuvant cisplatin, $\mathrm{N}$-2 (chloroethyl)- $\mathrm{N}$-cyclohexyl- $N$-nitrosourea, and vincristine. The best 5-year progression-free survival rates for children treated with craniospinal irradiation alone are between $55 \%$ and $65 \%$. Most patients in our study did not receive adjuvant chemotherapy after radiation, and the 5-year progression-free survival appears to be consistent with that reported for children treated similarly after diagnosis (with craniospinal radiation alone).

Sixteen patients were designated poor risk at diagnosis, however, only 3 of these patients had $\mathrm{M}+$ disease. A recent report by the Children's Cancer Group suggests that the presence of metastatic disease is a major prognostic factor in children older than 3 years (Zeltzer et al., 1999). They found that 5 -year progression-free survival was $70 \%$ in M0, $57 \%$ in M1, and $40 \%$ in M2 patients. Packer et al. 


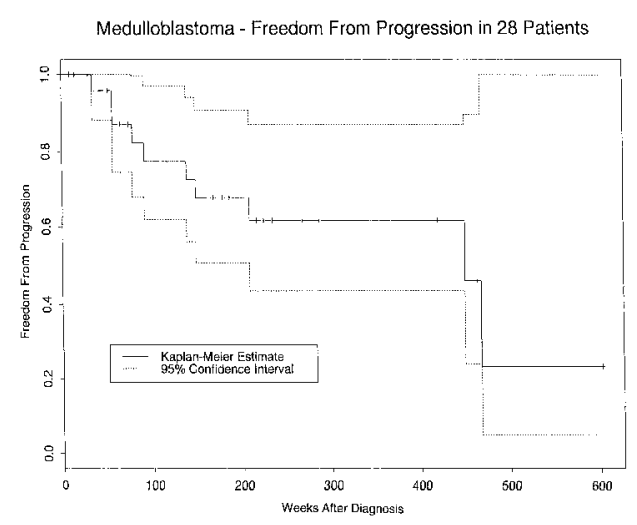

Fig. 2. Kaplan-Meier estimate of freedom from progression for adult medulloblastoma. Time to progression was measured from date of diagnosis until date of confirmed recurrence after resection and radiotherapy with or without adjuvant chemotherapy.

(1994) additionally found that poor risk patients with metastatic disease at diagnosis compared with those patients with localized disease alone had a significantly worse survival. The administration of adjuvant chemotherapy after craniospinal irradiation to poor-risk adult patients did not appear to improve survival in either this or the larger multicenter study reported by Carrie et al. (1994). If staging based solely on the presence of metastasis is used, then our cohort of patients was composed essentially of a group of standard-risk patients in that 25 of 28 were $\mathrm{M} 0$, although the lack of formal radiographic review may have confounded our results. Our findings suggest that the administration of adjuvant chemotherapy to patients designated as poor risk based on extent of residual local disease after resection, rather than metastasis, may not improve progression-free or overall survival. Certainly this question can only be answered by a prospective study.

The marked difference seen between the overall and progression-free 5-year survival rates appears to represent successful salvage of relapsed patients. Survival after recurrence in children has typically been poor. Because of the variety of therapies delivered at recurrence and the number of patients still living after recurrence, we are unable to comment on response to any particular therapy at recurrence or on median survival after recurrence. The high overall survival, despite recurrence, suggests that adult medulloblastoma remains responsive to therapy after first recurrence. Despite the small numbers of patients, salvage treatment with chemotherapy appears to have a role in recurrent disease in that several patients had durable responses after recurrence.
Analysis of other clinical variables did not allow us to define prognostic value of any clinical feature among our 28 patients. Le et al. (1997) reported that age was not significant as a prognostic variable in univariate analysis, but became significant in multivariate analysis such that older age became favorable in men with localized disease at diagnosis. Because of our limited population size, we did not attempt to delineate prognostic variables by multivariate analysis.

Other patient characteristics-such as female sex, localized extent of disease, and gross total resectionpreviously reported to reach significance as favorable prognostic variables among the pediatric population, were not found to reach significance in our study. Our cohort had a slight male predominance; however, male sex was not a poor prognostic factor as it appears to be in children (Prados et al., 1996; Weil et al., 1998). Prados et al. (1996) reported on a mixed group of adults and children for which the 5-year survival for females was $92 \%$ and for males was $40 \%$. The difference in survival in their patients may reflect biologic differences between children and adults. Although 2 of the 3 patients with metastatic disease had a short survival, the prognostic role of metastatic disease at diagnosis cannot be defined due to the small population size. A higher proportion of desmoplastic medulloblastoma occurs in adults (Giordana et al., 1997) than in children. Desmoplastic medulloblastoma has been suggested by other groups to have a better prognosis, but in our series the 2 histologic types had similar outcomes.

The pattern of failure seen in the larger group of 57 patients exhibits an interesting observation. Seven of the $32(22 \%)$ recurrences occurred solely as extraneural, systemic metastasis, and in 5 of the $32(16 \%)$ cases systemic metastasis occurred in tandem with CNS recurrence. This degree of extraneural metastasis is in excess of that reported for children with medulloblastoma (Packer et al., 1994). The extraneural metastasis was not associated with placement of a ventriculoperitoneal shunt as only 1 of 12 affected patients had such a device. This pattern of failure may further represent a biologic difference between the tumor found in adults versus that found in children.

The optimal treatment of adult medulloblastoma remains unclear. The practice of using staging data developed for childhood medulloblastoma to determine risk for recurrence and poorer prognosis may not be valid in the adult population. The current standard of care remains maximal surgical resection of the primary neoplasm followed by craniospinal irradiation. The optimal use of adjuvant chemotherapy remains unclear, but may be best reserved for patients with CNS or extraneural metastasis at diagnosis.

\section{References}

Bloom, H.J.G., and Bessel, E.M. (1990) Medulloblastoma in adults: A review of 47 patients treated between 1952 and 1981. Int. J. Radiat. Oncol. Biol. Phys. 18, 763-772.

Carrie, C., Lasset, C., Alapetite, C., Haie-Meder, C., Hoffstetter, S., Demaille, M.C., Kerr, C., Wagner, J.P., Lagrange, J.L., Maire, J.P., Seng, S., Man, Y., Murraciole, X., and Pinto, N. (1994) Multivariate analysis of prognostic factors in adult patients with medulloblastoma: Retrospective study of 156 patients. Cancer 74, 2352-2360.

Giordana, M.T., Cavalla, P., Dutto, A., Borsotti, L., Chio, A., and Schiffer, D. (1997) Is medulloblastoma the same tumor in children and adults? J. Neurooncol. 35, 169-176.

Giordana, M.T., Schiffer, P., Lanotte, M., Girardi, P., and Chio, A. (1999) Epi- 
demiology of adult medulloblastoma. Int. J. Cancer 80, 689-692.

Haie, C., Schlienger, M., Constans, J.P., Meder J.F., Reynaud, A., and Ghenim, C. (1985) Results of radiation treatment of medulloblastoma in adults. Int. J. Radiat. Oncol. Biol. Phys. 11, 2051-2056.

Le, Q.T., Weil, M.D., Wara, W.M., Lamborn, K.R., Prados, M.D., Edwards, M.S., and Gutin, P.H. (1997) Adult medulloblastoma: An analysis of survival and prognostic factors. Cancer J. Sci. Am. 3, 238-245.

Noel, G., and Merrer, J. (1997) Medulloblastoma in adults: Val-de-Grace Hospital experience (1975-1994) and literature review [in French]. Cancer Radiother. 1, 60-67.

Packer, R.J., Sutton, L.N., Elterman, R., Lange, B., Goldwein, J., Nicholson, H.S., Mulne, L., Boyett, J., D'Angio, G., Wechsler-Jentzsch, K., Reaman, G., Cohem, B.H., Bruce, D.A., Rorke, L.B., Molloy, P., Ryan, J., LaFond, D., Evans, A.E., and Schut, L. (1994) Outcome for children with medulloblastoma treated with radiation and cisplatin, CCNU, and vincristine chemotherapy. J. Neurosurg. 81, 690-698.

Prados, M.D., Wara, W., Edwards, M.S., Ater, J., Rabbit, J., Lamborn, K.,
Davis, R., and Levin, V.A. (1996) Treatment of high-risk medulloblastoma and other primitive neuroectodermal tumors with reduced dose craniospinal radiation therapy and multi-agent nitrosourea-based chemotherapy. Pediatr. Neurosurg. 25, 174-181.

Polednak, A.P., and Flannery, J.T. (1995) Brain, other central nervous system, and eye cancer. Cancer 75 (Suppl. 1), 330-337.

Weil, M.D., Lamborn K., Edwards, M.S.B., and Wara W.M. (1998) Influence of a child's sex on medulloblastoma outcome. JAMA 279, 1474-1476.

Wolff, J.E., Huettermann, U., Ritter, J., Straeter, R., Palm, D., Ruebe, C., Kraehling, K.H., and Jurgens, H. (1998) Medulloblastoma: Experience of a single institution. Klin. Padiatr. 210, 234-238.

Zeltzer, P.M., Boyett, J.M., Finlay, J.T., Albright, A.L., Rorke, L.B., Mulstein, J.M., Allen, J.C., Stevens, K.R., Stanley, P., Li, H., Wisoff, J.H., Geyer, J.R., McGuire-Cullen, P., Stehbens, J.A., Shurin, S.B., and Packer, R.J. (1999) Metastasis stage, adjuvant therapy, and residual tumor are prognostic factors for medulloblastoma in children: Conclusions from the Children's Cancer Group 921 randomized phase III study. J. Clin. Oncol. 17, 832-845. 